\title{
Relationship of cerebral microbleeds to inflammatory marker levels
}

\author{
Qiao-Li Lu', Chen Li', Ying Song ${ }^{1}$, Liang Wang ${ }^{2}$, Zhi-Rong Jia ${ }^{3}$ \\ ${ }^{1}$ Department of Neurology, Tianjin 5th Center Hospital, Tianjin 300450, China. \\ ${ }^{2}$ Department of Neurosurgery, Tianjin 5th Center Hospital, Tianjin 300450, China. \\ ${ }^{3}$ Department of Neurology, Peking University First Hospital, Beijing 100034, China.
}

Correspondence to: Dr. Chen Li, Department of Neurology, Tianjin 5th Center Hospital, 41 Zhejiang Road, Tanggu, Tianjin 300450, China. E-mail: lichenokk@163.com

How to cite this article: Lu QL, Li C, Song Y, Wang L, Jia ZR. Relation of cerebral microbleeds to inflammatory marker levels. Neuroimmunol Neuroinflammation 2017;4:145-51.

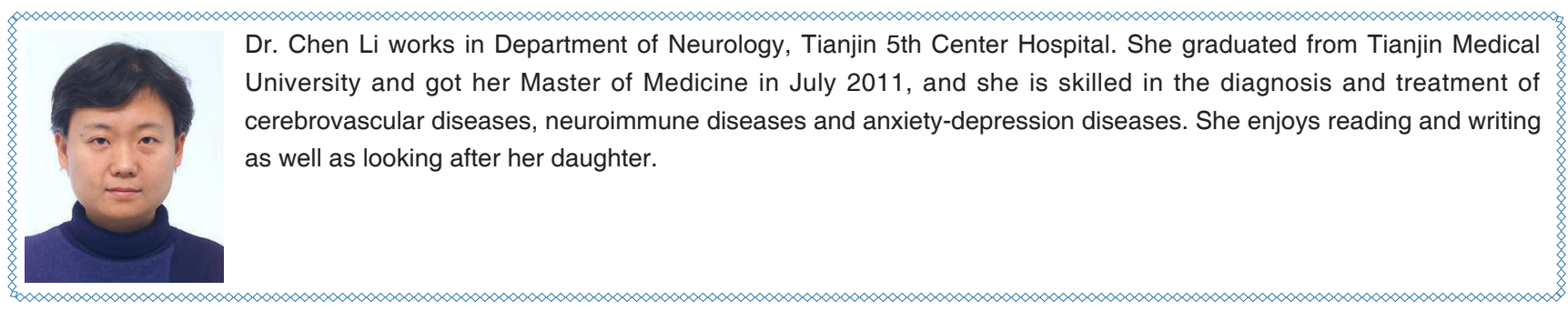

Article history:

Received: 24-02-2017

Accepted: 10-05-2017

Published: 08-08-2017

\section{Key words:}

Cerebral microbleed, traditional risk factor, inflammatory marker level, susceptibility-weighted imaging

\begin{abstract}
ABSTRAC T
Aim: The purpose of this study is to investigate the incidence, distribution and risk factors of cerebral microbleeds (CMBs) and the relation between CMBs and inflammation in ischemic cerebrovascular disease. Methods: Two hundred and one patients without acute infarction or transient ischemic attack were enrolled. The presence and number of $\mathrm{CMB}$ were assessed on susceptibility-weighted imaging. The traditional risk factors of CMB were recorded. Levels of high-sensitivity C-reactive protein (hs-CRP), interleukin-6 (IL-6), and matrix metalloproteinase-9 (MMP-9) were tested. Logistic regression analyses were used for multiple-factor analysis of risk factors of CMB. Results: Of the 201 patients, 49 (24.38\%) had CMB. Multivariate logistic regression analyses showed that the age, the prevalence of hypertension, silent lacunar infarction, white matter lesion, Montreal Cognitive Assessment Score, the using rate of antithrombotic drugs and levels of hs-CRP, IL-6, MMP-9 were the risk factors for CMB. After adjustments for traditional risk factors, inflammatory marker levels remained to be associated with CMBs. The adjusted odd ratios of hs-CRP, IL-6 and MMP-9 were 1.745 (1.342-2.270), 1.223 (1.018-1.533) and 1.284 (1.082-1.423), respectively. Furthermore, inflammatory marker levels were the risk factor for deep or infratentorial CMBs and lobar CMBs. Conclusion: The age, prevalence of hypertension, silent lacunar infarction, white matter lesion, MoCA Score, the using rate of antithrombotic drugs and serum hs-CRP, IL-6, and MMP-9 levels were the independent risk factors for CMBs.
\end{abstract}

(i) (2) This is an open access article distributed under the terms of the Creative Commons AttributionNonCommercial-ShareAlike 3.0 License, which allows others to remix, tweak, and build upon the work non-commercially, as long as the author is credited and the new creations are licensed under the identical terms.

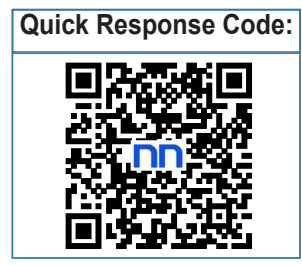




\section{INTRODUCTION}

Cerebral microbleeds (CMBs) are designated as a microvascular disease which has no related symptoms and physical signs..$^{[1]} \mathrm{CMBs}$ has a round or ovoid signal of hypointensity $(\mathrm{d}<10 \mathrm{~mm})$ in $\mathrm{T}^{*}$ Gradient-Recall Echo (GRE) and susceptibilityweighted imaging (SWI) sequences. ${ }^{[2]}$ Previous studies suggested that damage of cerebral microvasculature resulted from hypertensive vasculopathy, cerebral amyloid angiopathy (CAA) and ischemic brain damage. ${ }^{[3]} \mathrm{CMBs}$ are associated with hypertensive vasculopathy tends to occur in basal ganglia, whereas advanced CAA is associated with a lobar distribution. ${ }^{[1]}$ CMBs exists widely in patients of ischemic and hemorrhagic stroke, but also in normal elderly individuals. ${ }^{[1]}$ Previous studies have shown that stroke patients combined with CMBs have a significantly higher risk of recurrence. ${ }^{[3]}$ The presence of CMBs was also independently associated with cognitive functions. ${ }^{[4]}$ Multiple CMBs always indicate serious microvascular lesion which will increase risk of rebleeding. ${ }^{[5]}$ At the same time, patients with CMBs had a higher risk of spontaneous hemorrhage while receiving anticoagulation and thrombolytic therapy. ${ }^{[5]}$ CMBs are related to antiplatelet-related intracerebral hemorrhage. ${ }^{[6]}$ Recently, an individual participant meta-analysis showed that C-reactive protein concentration was associated with the risk of coronary heart disease and ischemic stroke. ${ }^{[7]}$ Inflammatory marker interleukin-6 (IL-6) retained a significant association with coronary heart disease and ischemic stroke..$^{[8]} \mathrm{A}$ review revealed that matrix metalloproteinase-9 (MMP-9) levels were significantly correlated with severity of stroke, larger infarct volume and worse functional outcome. ${ }^{[9]}$ For the above reason, we sought to choose high-sensitivity C-reactive protein (hs-CRP), IL-6 and MMP-9 to investigate the relationship between inflammatory marker levels and CMBs so as to prevent CMBs from developing.

\section{METHODS}

\section{Population}

Between June 2013 and March 2015, we recruited 286 participants with a age was greater than 45 years of age from both outpatient and in-patient services of the Department of Neurology, Peking University Binhai Hospital. In this study, the majority of the patients were without acute or transient neurologic impairment. We excluded 18 patients with a history of intracerebral hemorrhage and 9 patients with a history of traumatic brain injury. In addition, infectious diseases $(n=12)$, collagen disease $(n=8)$, malignant disease $(n=4)$ and patients with severe co-morbid medical conditions $(n=10)$ and those using anti-inflammatory medications $(n=13)$ were also excluded. We further excluded patients whose hs-CRP levels were higher than $8 \mathrm{mg} / \mathrm{L}(n=11)$. Finally, 201 patients were included in the analysis. We divided the patients into 2 groups based on SWI, CMBs group $(n=49)$ and no CMBs group ( $n=152$ ), CMBs group was further divided into deep or infratentorial CMBs group $(n=30)$ and lobar CMBs group $(n=19)$ based on CMBs location.

\section{Risk factors assessment}

The data including age, gender, body weight, height and medical history of patients such as smoking, alcohol intake, heart disease, blood pressure, blood glucose, serum lipid and the use of antithrombotic drugs were recorded for analysis. Hypertension was defined as blood pressure $\geq 140 / 90 \mathrm{mmHg}$ on measurements taken on at least two occasions, or patients with a history of hypertension and using antihypertensive drug. Hyperlipidemia was defined as triglyceride level $\geq 1.7 \mathrm{mmol} / \mathrm{L}$; low-density lipoprotein cholesterol level $\geq 3.4 \mathrm{mmol} / \mathrm{L}$; total cholesterol level $\geq 5.72 \mathrm{mmol} / \mathrm{L}$, or use of cholesterol-lowering therapy. Diabetes was defined as fasting blood-glucose $\geq 7.0 \mathrm{~mol} / \mathrm{L}$ and/or postprandial blood sugar $\geq 11.1 \mathrm{~mol} / \mathrm{L}$, or use of antidiabetic therapy. Body mass index was defined as weight $[\mathrm{kg}] /$ height $[\mathrm{m}]^{2}$. Smoking was defined as ever smoking but giving up and current smoking. Habitual alcohol intake was defined as alcohol drinking more than $20 \mathrm{~g} /$ day antiplatelet drugs and anticoagulant drugs are regarded as antithrombotic drugs. Montreal Cognitive Assessment Scores were used to assess cognitive function.

\section{Magnetic resonance imaging parameters and diagnosis of $\mathrm{CMB}$}

Brain magnetic resonance imaging (MRI) was performed with a 3.0-tesla MR unit (Achieva; Philips). Susceptibility-weighted imaging $(\mathrm{TR}=17 \mathrm{~ms}$, $\mathrm{TE}$ = $24 \mathrm{~ms}, 10-\mathrm{mm}$-thick slices with $5-\mathrm{mm}$ spacing) was included in the routine protocol. All the images were compared and analyzed on the workstation by trained and experienced radiologist and neurologist who were blinded to clinical information at the same time according to the Microbleed Anatomical Rating Scale MARS. ${ }^{[10]} \mathrm{A}$ single CMB was defined as a small, round, or ovoid hypointensity of $<10 \mathrm{~mm}$ in diameter, evident on T2* GRE or SWI MRI sequences. ${ }^{[2]}$ Microbleed mimics (e.g. vessels, mineralization, airbone interfaces, partial volume artifact at the edges of the cerebellum) were excluded. ${ }^{[10]}$ CMBs were divided into lobar microbleeds (cortex, subcortex, and white matter) and deep or infratentorial 
microbleeds (basal ganglia, thalamus, brainstem and cerebellum) ${ }^{\left[{ }^{[1]}\right.}$ Further, we made categories for "strictly lobar microbleeds" (persons with $\geq 1$ new microbleeds restricted to a lobar location) and "deep or infratentorial microbleeds" (persons with $\geq 1$ new microbleeds in a deep or infratentorial location with or without lobar microbleeds). ${ }^{[12]}$ Silent lacunar infarction was defined as an area of low signal intensity on T1-weighted images with corresponding high signal intensity on T2-weighted images, whose diameter was $>3 \mathrm{~mm}$ and $<15 \mathrm{~mm}$ (e.g. dilated perivascular space). ${ }^{[13]}$ Diagnostic criteria of white matter lesion: periventricular white matter lesions (WMLs) were scored according to the following patterns: no lesions (0 points); pencil-like or cap-like thin lesions (1 point); smooth haloes at lesion site (2 points); and irregular periventricular high signals extending to deep WM (3 points). Deep WMLs were scored according to the following patterns: no lesions (0 points); punctate separate lesions ( 1 point); fused lesions (2 points); and large fused lesions ( 3 points). The total score was obtained by adding the periventricular and deep WMLs scales together. ${ }^{[14]}$

\section{Measurement of inflammatory markers}

Blood was drawn with minimally traumatic venipuncture for measurement of serum inflammatory markers. Blood were centrifuged by $3,000 \mathrm{~g}$ for $15 \mathrm{~min}$ at $4{ }^{\circ} \mathrm{C}$, and then aliquots were stored at $-70{ }^{\circ} \mathrm{C}$. Serum hs-CRP was measured by latex turbidimetric immunoassay with a sensitivity of $0.01 \mathrm{mg} / \mathrm{L}$. Serum IL-6 and MMP-9 were measured by enzyme-linked immunosorbent assay (High Sensitivity Quantikine kit; R\&D System) with a sensitivity of $0.01 \mathrm{pg} / \mathrm{mL}$. Serum MMP-9 was also measured by enzyme-linked immunosorbent assay (High Sensitivity Quantikine kit; R\&D System). The detectable limit for Serum IL-6 and MMP-9 were $0.01 \mathrm{pg} / \mathrm{mL}$ and $0.01 \mathrm{ng} / \mathrm{mL}$, respectively.

\section{Statistical analysis}

Data was analyzed using SPSS 19.0. Measurement data was described as mean \pm standard deviation (SD). Enumeration data was described as number (\%). $T$-test and one-way analysis of variance was used for comparisons of continuous variables. We used $\chi^{2}$ test for enumeration data. Skewed distribution data was described as (Media and Q1-Q3). Kruskal-Wallis test followed by the Mann-Whitney $U$ test were used for comparisons between groups. Multivariate logistic regression analyses were used for calculation of odds ratio, in which logarithmically transformed values of inflammatory markers were used. The results are shown as the odd ratios (OR) with $95 \%$ confidence interval $(\mathrm{Cl})$. Probability values were 2-tailed, and values of $P<0.05$ were considered significant.

\section{RESULTS}

\section{CMB distributional characteristics}

Of the patients, $49(24.38 \%)$ had CMBs. The spatial distribution of the CMB number and location was as follows: deep or infratentorial, 166 in 30 patients (61.22\%); lobar, 88 in 19 patients (38.78\%).

\section{Relations between CMBs and traditional risk factors}

The baseline characteristics of the patients in this study are shown in Tables 1 and 2. There were significant differences in the traditional risk factors such as age, the prevalence of hypertension, coronary heart disease, silent lacunar infarction (SLI) and WMLs,

Table 1: Comparison of baseline characteristics between CMB group and no CMB group

\begin{tabular}{|c|c|c|c|}
\hline \multirow{2}{*}{ Characteristics } & CMB group & No CMB group & \multirow{2}{*}{$P$} \\
\hline & $(n=49)$ & $(n=152)$ & \\
\hline Age (years) & $68.61 \pm 7.76^{\dagger}$ & $61.76 \pm 11.06$ & 0.011 \\
\hline Male $(n, \%)$ & 29 (59.18) & 79 (51.97) & 0.483 \\
\hline BMI $\left(\mathrm{kg} / \mathrm{m}^{2}\right)$ & $26.89 \pm 2.96$ & $25.63 \pm 2.76$ & 0.068 \\
\hline Smoking $(n, \%)$ & $21(42.85)$ & $37(47.37)$ & 0.642 \\
\hline Alcohol intake $(n, \%)$ & $26(53.06)$ & $74(48.68)$ & 0.494 \\
\hline Hypertension $(n, \%)$ & $33(67.34)^{\ddagger}$ & $61(40.13)$ & 0.001 \\
\hline $\mathrm{DM}(n, \%)$ & $20(40.81)$ & $60(39.47)$ & 0.868 \\
\hline $\operatorname{HLP}(n, \%)$ & $21(42.85)$ & $57(37.50)$ & 0.506 \\
\hline $\mathrm{CHD}(n, \%)$ & $29(59.18)^{\dagger}$ & $64(42.10)$ & 0.048 \\
\hline $\operatorname{SLI}(n, \%)$ & $35(71.42)^{\ddagger}$ & $47(30.92)$ & 0.001 \\
\hline WML (M, Q1-Q3) & $5(4-6)^{\ddagger}$ & $2(1-4)$ & 0.000 \\
\hline Antithrombotic drugs $(n, \%)$ & $27(55.10)^{\dagger}$ & 54 (35.53) & 0.019 \\
\hline MoCA Score & $24.45 \pm 0.29^{\ddagger}$ & $26.62 \pm 0.21$ & 0.006 \\
\hline hs-CRP [mg/L (M, Q1-Q3)] & $6.83(5.91-9.73)^{\ddagger}$ & $3.20(2.10-5.34)$ & 0.000 \\
\hline IL-6 [pg/mL (M, Q1-Q3)] & $8.23(6.68-12.20)^{\ddagger}$ & 5.59 (3.72-7.79) & 0.000 \\
\hline MMP-9 [ng/mL (M, Q1-Q3)] & $15.98(13.65-18.46)^{\ddagger}$ & $11.66(7.78-15.77)$ & 0.001 \\
\hline
\end{tabular}

${ }^{\dagger} P<0.05,{ }^{\ddagger} P<0.01$, compared with the no CMB group. CMB: cerebral microbleeds; BMI: body mass index; DM: diabetes mellitus; HLP: hyperlipemia; CHD: coronary heart disease; SLI: silent lacunar infarction; hs-CRP: high-sensitivity C-reactive protein; IL: interleukin; MMP: matrix metalloproteinase 
MoCA Scores or the using rate of antithrombotic drugs between the CMB group and no CMB group $(P<0.05$, Table 1). Furthermore, we also observed similar associations between traditional risk factors and deep or infratentorial CMBs [Table 2]. Similarly, there were significant differences such as age, or the prevalence of alcohol intake, SLI and WML between the lobar CMB and no CMB group $(P<0.05$, Table 2$)$. Compared with the lobar CMB group, there was higher prevalence of hypertension and lower alcohol intake in deep or infratentorial CMB group [Table 2]. On logistic regression analysis, the association between traditional risk factors and CMBs are summarized in Table 3. The ORs (per 1SD increase; $95 \% \mathrm{Cl}$ ) of age, the prevalence of hypertension, SLI and WML, MoCA Score and the using rate of antithrombotic drugs were 1.666 (1.062-2.632), 2.634 (1.067-4.767), 2.345 (1.056-5.226), 1.717 (1.132-2.603), 1.198 (1.023-2.268) and 1.234 (1.116-2.584), respectively. In addition, the association between traditional risk factors and deep or infratentorial CMBs were also stronger [Table 3]. However, for strictly lobar CMB, the only independent risk factor was age and the prevalence of alcohol intake [Table 3].
Relations between CMBs and inflammatory marker levels

We observed that serum hs-CRP, IL-6 and MMP-9 levels (median: $6.83,8.23,15.98$, respectively) in the CMB group were higher than in those (median: 3.20 , $5.59,11.66$, respectively) without CMB group [Table 1]. Similarly, we observed that hs-CRP, IL-6 and MMP-9 levels were higher in the deep or infratentorial CMB group (median: 7.01, 9.31, 17.63, respectively) or lobar CMB group (median: 6.82, 7.98, 15.84, respectively) than in those without [Table 2]. There was no significant difference between the deep or infratentorial CMB group and lobar CMB group. Connections between $\mathrm{CMB}$ and inflammatory markers are summarized in Table 4. Logistic regression analysis showed that each 1SD-increase in each inflammatory marker level was significantly associated with the presence of $\mathrm{CMB}$ after adjustment for age and sex (Model 1). After adjustment for age, sex, BMI, smoking, alcohol intake, hypertension, diabetes, hyperlipidemia, coronary heart disease, the useful of antithrombotic drugs and the presence of SLI and WML, inflammatory marker level remained to be associated with CMB (Model 2).

The adjusted ORs of hs-CRP, IL-6 and MMP-9

Table 2: Comparison of baseline characteristics between deep or infratentorial CMB group and lobar CMB group

\begin{tabular}{|c|c|c|c|}
\hline \multirow{2}{*}{ Characteristics } & Deep or infratentorial CMB group & Lobar CMB group & No CMB group \\
\hline & $(n=30)$ & $(n=19)$ & $(n=152)$ \\
\hline Age (years) & $69.77 \pm 7.76^{\ddagger}$ & $67.54 \pm 7.88^{\ddagger}$ & $61.76 \pm 11.06$ \\
\hline Male $(n, \%)$ & 19 (63.33) & 10 (52.63) & 79 (51.97) \\
\hline BMI $\left(\mathrm{kg} / \mathrm{m}^{2}\right)$ & $26.65 \pm 3.35$ & $25.39 \pm 2.89$ & $25.63 \pm 2.76$ \\
\hline Smoking $(n, \%)$ & $11(36.67)$ & $9(47.37)$ & $37(47.37)$ \\
\hline Alcohol intake $(n, \%)$ & $12(40.00)^{*}$ & $14(73.68)^{\dagger}$ & $74(48.68)$ \\
\hline Hypertension $(n, \%)$ & $24(80.00)^{\ddagger \star}$ & $9(47.37)$ & $61(40.13)$ \\
\hline $\mathrm{DM}(n, \%)$ & $13(43.33)$ & $7(36.84)$ & $60(39.47)$ \\
\hline $\operatorname{HLP}(n, \%)$ & $14(46.67)$ & $7(36.84)$ & $57(37.50)$ \\
\hline $\mathrm{CHD}(n, \%)$ & $20(66.67)^{\dagger}$ & $10(52.63)$ & $64(42.10)$ \\
\hline $\operatorname{SLI}(n, \%)$ & $24(80.00)^{\ddagger}$ & $11(57.89)^{\dagger}$ & $47(30.92)$ \\
\hline WML (M, Q1-Q3) & $5.5(4-6)^{\ddagger}$ & $5(4-6)^{\dagger^{\prime}}$ & $2(1-4)$ \\
\hline Antithrombotic drugs ( $n, \%)$ & $18(60.00)^{\dagger}$ & 9 (47.37) & $54(35.53)$ \\
\hline MoCA Score & $24.20 \pm 1.56^{\ddagger}$ & $24.58 \pm 1.07^{\dagger}$ & $26.62 \pm 0.21$ \\
\hline hs-CRP [mg/L (M, Q1-Q3)] & $7.01(5.23-9.93)^{\ddagger}$ & $6.82(5.67-9.80)^{\ddagger}$ & $3.20(2.10-5.34)$ \\
\hline IL-6 [pg/mL (M, Q1-Q3)] & $9.31(8.15-13.82)^{\ddagger}$ & $7.98(5.80-10.81)^{\ddagger}$ & $5.59(3.72-7.79)$ \\
\hline MMP-9 [ng/mL (M, Q1-Q3)] & $17.63(14.02-18.91)^{\ddagger}$ & $15.84(13.02-16.74)^{\ddagger}$ & $11.66(7.78-15.77)$ \\
\hline
\end{tabular}

${ }^{\dagger} P<0.05$, ${ }^{\ddagger} P<0.01$, compared with the no CMB group; ${ }^{*} P<0.05$, ${ }^{* *} P<0.01$, compared with the lobar CMB group. CMB: cerebra microbleeds; BMI: body mass index; DM: diabetes mellitus; HLP: hyperlipemia; CHD: coronary heart disease; SLI: silent lacunar infarction; hs-CRP: high-sensitivity C-reactive protein; IL: interleukin; MMP: matrix metalloproteinase

Table 3: OR (95\% Cl) for CMB status per 1SD increase in traditional risk factors

\begin{tabular}{lccc}
\hline Variable & CMB OR (95\% CI) & $\begin{array}{c}\text { Deep or infratentorial } \\
\text { CMB OR (95\% CI) }\end{array}$ & $\begin{array}{c}\text { Lobar } \\
\text { CMB OR (95\% CI) }\end{array}$ \\
\hline Age & $1.666(1.062-2.632)^{\dagger}$ & $1.773(1.172-2.641)^{\dagger}$ & $1.436(1.134-2.442)^{\dagger}$ \\
Hypertension & $2.634(1.067-4.767)^{\dagger}$ & $3.875(1.795-5.955)^{\ddagger}$ & \\
SLI & $2.345(1.056-5.226)^{\dagger}$ & $2.438(1.226-4.750)^{\ddagger}$ & \\
WML & $1.717(1.132-2.603)^{\dagger}$ & $1.850(1.291-2.643)^{\dagger}$ & \\
MoCA Score & $1.198(1.023-2.268)^{\dagger}$ & $1.230(1.100-2.448)^{\dagger}$ & \\
Antithrombotic drugs & $1.234(1.116-2.584)^{\dagger}$ & $1.290(1.001-2.229)^{\ddagger}$ & $1.023(1.008-1.058)^{\dagger}$ \\
Alcohol intake & & & \\
\hline
\end{tabular}

${ }^{\dagger} P<0.05,{ }^{\ddagger} P<0.01$, compared with the no CMB group. SD: standard deviation; Cl: confidence interval; OR: odd ratios; CMB: cerebral microbleeds; SLI: silent lacunar infarction; WML: white matter lesion 
Table 4: OR (95\% Cl) for CMB status per 1SD increase in inflammation factors (values were log transformed for analysis)

\begin{tabular}{|c|c|c|c|c|c|c|}
\hline \multirow[b]{2}{*}{ Variable } & \multicolumn{2}{|c|}{ CMB } & \multicolumn{2}{|c|}{ Deep or infratentorial CMB } & \multicolumn{2}{|c|}{ Lobar CMB } \\
\hline & $\begin{array}{c}\text { Model } 1 \\
\text { OR }(95 \% \mathrm{Cl})\end{array}$ & $\begin{array}{c}\text { Model } 2 \\
\text { OR }(95 \% \mathrm{Cl})\end{array}$ & $\begin{array}{c}\text { Model } 1 \\
\text { OR }(95 \% \mathrm{Cl})\end{array}$ & $\begin{array}{c}\text { Model } 2 \\
\text { OR }(95 \% \text { Cl) }\end{array}$ & $\begin{array}{c}\text { Model } 1 \\
\text { OR }(95 \% \mathrm{Cl})\end{array}$ & $\begin{array}{c}\text { Model } 2 \\
\text { OR }(95 \% \mathrm{Cl})\end{array}$ \\
\hline hs-CRP & $1.852(1.435-2.391)^{\ddagger}$ & $1.745(1.342-2.270)^{\ddagger}$ & $2.372(1.680-3.262)^{\ddagger}$ & $2.302(1.520-3.482)^{\ddagger}$ & $1.578(1.043-2.337)^{\ddagger}$ & $1.534(1.009-2.259)^{\ddagger}$ \\
\hline IL-6 & $1.469(1.204-1.792)^{\dagger}$ & $1.223(1.018-1.533)^{\ddagger}$ & $1.480(1.102-2.492)^{\ddagger}$ & $1.334(1.008-3.660)^{\dagger}$ & $1.422(1.001-2.678)^{\ddagger}$ & $1.508(1.097-3.428)^{\dagger}$ \\
\hline MMP-9 & $1.397(1.196-1.632)^{\ddagger}$ & $1.284(1.082-1.423)^{\ddagger}$ & $1.420(1.241-1.799)^{\ddagger}$ & $1.287(1.145-1.599)^{\ddagger}$ & $1.293(1.142-2.423)^{\ddagger}$ & $1.242(1.178-1.409)^{\ddagger}$ \\
\hline
\end{tabular}

${ }^{\dagger} P<0.05,{ }^{\ddagger} P<0.01$, compared with the no CMB group. Model 1: adjusted for age and sex; Model 2: adjusted for age, sex, body mass index, smoking, alcohol intake, hypertension, diabetes, hyperlipidemia, coronary heart disease, the presence of silent lacunar infarction and white matter lesion and antithrombotic drugs. CMB: cerebral microbleeds; SD: standard deviation; Cl: confidence interval; OR: odd ratios; hs-CRP: high-sensitivity C-reactive protein; IL: interleukin; MMP: matrix metalloproteinase

were $1.745(1.342-2.270), 1.223(1.018-1.533)$ and 1.284 (1.082-1.423), respectively. Furthermore, the associations between inflammatory marker level and deep or infratentorial CMB or lobar CMB remained significant after adjustment for age and sex (Model 1) and after additional adjustment for the traditional risk factors (Model 2). The adjusted ORs of hs-CRP, IL-6 and MMP-9 for the presence of deep or infratentorial CMB were 2.302 (1.520-3.482), 1.334 (1.008-3.660) and 1.287 (1.145-1.599), respectively. For the presence of lobar CMB, the adjusted ORs of hs-CRP, IL-6 and MMP-9 were 1.534 (1.009-2.259), 1.508 (1.097-3.428) and 1.242 (1.178-1.409), respectively.

\section{DISCUSSION}

The prevalence of CMBs varies from different population. A meta-analyze found that incidence of CMBs was $44 \%$ in ischemic stroke, $83 \%$ in intracerebral hemorrhage, and $5-6 \%$ in healthy adults. ${ }^{[15]}$ In this study, the incidence of CMBs was $24.38 \%, 30$ patients $(61.22 \%)$ had deep or infratentorial CMB, 19 patients $(38.78 \%)$ had lobar $\mathrm{CMB}$. The prevalence of CMB in our study was higher than that in healthy adults, but, lower than that in ischemic stroke. The first possible reason was the patients who participated without acute infarction or transient ischemic attack. The second possible reason was the use of $S W I$, it may increase the prevalence and number of CMB detected, compared with T2 GRE.

On assessment, CMB are often categorized according to location, separating strictly lobar CMB from deep or infratentorial CMB. Lobar CMB represents cerebral amyloid angiopathy, but, deep and infratentorial CMB is attributed to hypertensive arteriopathy. ${ }^{[16]}$ Poels et al. ${ }^{[17]}$ found an association between atherosclerosis and deep or infratentorial $\mathrm{CMB}$ in individuals with uncontrolled hypertension. This may explain the theoretical assumption that deep or infratentorial $\mathrm{CMB}$ are attributed to hypertensive vasculopathy, whereas lobar CMB are a result of amyloid angiopathy. ${ }^{[18]}$ Several studies have demonstrated strictly that lobar CMB was associated with the apolipoprotein E4 allele and diastolic blood pressure. On the other hand, systolic blood pressure, pulse pressure, silent lacunar infarction, white matter hyperintensities and smoking were associated with CMB in the deep or infratentorial regions. ${ }^{[19]}$ In our study, hypertension, SLI, WML were associated with higher incidence of $\mathrm{CMB}$, and thus were strong risk factors of $\mathrm{CMB}$. The associations between traditional risk factors and deep or infratentorial CMB remained significant. Our results were consistent with previous studies. Shams et al. ${ }^{[16]}$ observed that patients with $\mathrm{CMB}$ were significantly aged, had hypertension, and had lower cognitive function. A number of studies have shown that age is an independent risk factor of CMB. Vernooij et al. ${ }^{[11]}$ found that the incidence of CMB increased with age from $17.8 \%$ in persons aged $60-69$ years to $38.3 \%$ in those over 80 years. There were significant differences in MoCA Scores and using antithrombotic drugs between the CMB group and no CMB group. Several studies reported an association between the presence of CMB and impaired cognitive function. ${ }^{[20,21]}$ Small vessel disease can be visualized as white matter hyperintensities and SLI but also as CMB on brain MRI. ${ }^{[21]} \mathrm{A}$ number of studies found that almost all the CMB patients had various degrees of WML and different numbers of SLI. Gregoire et al. ${ }^{[6]}$ found that CMB were more numerous and prevalent in antiplatelet users who developed symptomatic ICH compared with matched antiplatelet-users who did not develop $\mathrm{ICH}$. This data suggested a potential role for $\mathrm{CMB}$ as a risk factor for antiplatelet-associated $\mathrm{ICH}$. The relationship between alcohol intake and $\mathrm{CMB}$ needed to be further studied.

Our study showed that the levels of hs-CRP, IL-6, MMP9 in CMB group were significantly higher than those in no $C M B$ group. The regression analysis showed that inflammatory factors such as hs-CRP, IL-6 and MMP9 were the independent risk factors of CMB. Also, they were also the independent risk factors of deep or infratentorial $\mathrm{CMB}$ and lobar CMB. This suggested that 
$\mathrm{CMB}$ can be seen as the common downstream product of hypertensive vasculopathy and cerebral amyloid angiopathy pathways. ${ }^{[22]}$ In old patients, hemosiderin deposited around the capillaries, the hemosiderin leaking through expansile vascular clearance would accelerate the inflammation and result in small vessel disease and CMB. ${ }^{[23]}$ Previous studies found that levels of hs-CRP, IL-6, and IL-18 are related to both deep and lobar CMB. ${ }^{[22]}$ Hoshi et al. ${ }^{[13]}$ found that IL-6 could result in increase of hs-CRP as an important cytokine in inflammation, it can be a predictor of SLI. Koh et $a l .{ }^{[24]}$ found that the levels of MMP-9 and hsCRP were significantly higher in patients with $\mathrm{CMB}$ than in those without. Hemosiderin of brain microbleed is well known to activate MMP-9, increased MMP9 activity enhances inflammatory markers related to deterioration of neurological function in ischemic stroke. ${ }^{[24]}$ Pantoni et al. ${ }^{[25]}$ found that destruction of Blood Brain Barrie played an important role during the process of CMB. MMP-9 would destroy the blood-brain barrier (BBB) by degrading the extracellular matrix, leading to the increased permeability of BBB. ${ }^{[26]}$ The animal experiment also proved a close relationship between increased MMP-9 and chronic destruction of BBB. ${ }^{[27]} \mathrm{CMB}$ resulting from cerebral amyloid angiopathy could be confirmed as the presence of $\beta$-amyloid in the vessel wall, which activated microglia and $\mathrm{T}$ lymphocytes expressing heme oxygenase-1 activity and complement activation were prominent. The above studies supported that inflammatory reaction participate the dysfunction of vascular endothelium and destruction of BBB, finally leading to the occurrence of CMB. ${ }^{[28]}$

This study has several limitations. Firstly, the results of this research have limited effectiveness because of the relatively small number of cases, especially deep or infratentorial and lobar CMB group. More patients must be enrolled in a large clinical trial. Secondly, in the cross-sectional study, we did not recruit the general population as a control group. The relationships of $\mathrm{CMB}$ and inflammation in the general population remain unclear. Thirdly, we choose hs-CRP, IL-6 and MMP-9 to study the relationship between inflammatory marker and CMB. Other inflammatory factors need to be studied further to confirm the relationship between inflammatory marker and CMB.

In conclusion, our results demonstrated that $\mathrm{CMB}$ were closely related with the age, prevalence of hypertension, SLI, WML, MoCA Score, the using rate of antithrombotic drugs, levels of hs-CRP, IL-6, and MMP-9, suggesting a role of inflammatory processes in CMB. Patients with CMB have been consistently found to have an elevated risk of hemorrhagic stroke or an antithrombotic-related hemorrhagic complication. ${ }^{[29]}$ In the near future, we may reduce levels of circulating inflammatory markers to prevent CMB from happening.

\section{DECLARATIONS}

\section{Authors' contributions}

Substantially contributed to the general idea and design of the study: C. Li, Z.R. Jia

Took part in designing the protocol: Y. Song

Contributed to data collection and drafted the manuscript: Q.L. Lu

Helped with the data analysis: L. Wang

Read and approved the final manuscript: Q.L. Lu, C. Li, Y. Song, L. Wang, Z.R. Jia

\section{Acknowledgments}

We thank the laboratory and X-ray Department of Tianjin 5th Center Hospital for their secretarial assistance.

\section{Financial support and sponsorship}

This study was supported by a grant from Science and Technology Fund Project of Tianjin Health Bureau (No. 2013KZ021).

\section{Conflicts of interest}

The authors declare that they have no conflict of interest.

\section{Patient consent}

Informed consent was obtained from all individual participants included in the study.

\section{Ethics approval}

All procedures performed in the studies involving human participants were in accordance with the ethical standards of the institutional and/or national research committee and with the 1964 Helsinki Declaration and its later amendments or comparable ethical standards.

\section{REFERENCES}

1. Greenberg SM, Vernooij MW, Cordonnier C, Viswanathan A, AlShahi Salman R, Warach S, Launer LJ, Van Buchem MA, Breteler MM; Microbleed Study Group. Cerebralmicrobleeds: a guide to detection and interpretation. Lancet Neurol 2009;8:165-74.

2. Yates PA, Villemagne VL, Ellis KA, Desmond PM, Masters CL, Rowe CC. Cerebral microbleeds: a review of clinical, genetic, and neuroimaging associations. Front Neurol 2013;4:205.

3. Charidimou A, Kakar P, Fox Z, Werring DJ. Cerebral microbleeds and recurrent stroke risk: systematic review and meta-analysis of prospective ischemic stroke and transient ischemic attack cohorts. Stroke 2013;44:995-1001. 
4. Yamashiro K, Tanaka R, Okuma Y, Shimura H, Ueno Y, Miyamoto N, Urabe T, Hattori N. Cerebral microbleeds are associated with worse cognitive function in the nondemented elderly with small vessel disease. Cerebrovasc Dis Extra 2014;4:212-20.

5. Yamashiro K, Mori A, Shimada Y, Furuya T, Noda K, Urabe T, Hattori N, Okuma Y. Gradient echo T2*-weighted magnetic resonance imaging revealing cerebral microbleeds in a patient with microscopic polyangiitis complicated by cerebrovascular disease. $J$ Stroke Cerebrovasc Dis 2012;21:904.e7-9.

6. Gregoire SM, Jäger HR, Yousry TA, Kallis C, Brown MM, Werring DJ. Brain microbleeds as a potential risk factor for antiplatelet-related intracerebral haemorrhage: hospital-based, case-control study. $J$ Neurol Neurosurg Psychiatry 2010;81:679-84.

7. Emerging Risk Factors Collaboration, Kaptoge S, Di Angelantonio E, Lowe G, Pepys MB, Thompson SG, Collins R, Danesh J. $\mathrm{C}$-reactive protein concentration and risk of coronary heart disease, stroke, and mortality: an individual participant meta-analysis. Lancet 2010;375:132-40.

8. Patterson CC, Smith AE, Yarnell JW, Rumley A, Ben-Shlomo Y, Lowe GD. The associations of interleukin-6 (IL-6) and downstream inflammatory markers with risk of cardiovascular disease: the Caerphilly Study. Atherosclerosis 2010;209:551-7.

9. Ramos-Fernandez M, Bellolio MF, Stead LG. Matrix metalloproteinase-9 as a marker for acute ischemic stroke: a systematic review. J Stroke Cerebrovasc Dis 2011;20:47-54.

10. Gregoire SM, Chaudhary UJ, Brown MM, Yousry TA, Kallis C, Jäger HR, Werring DJ. The Microbleed Anatomical Rating Scale (MARS): reliability of a tool to map brain microbleeds. Neurology 2009;73:1759-66.

11. Vernooij MW, van der Lugt A, Ikram MA, Wielopolski PA, Niessen WJ, Hofman A, Krestin GP, Breteler MM. Prevalence and risk factors of cerebral microbleeds: the Rotterdam Scan Study. Neurology 2008;70:1208-11.

12. Goos JD, van der Flier WM, Knol DL, Pouwels P, Barkhof F, Wattjes MP. Clinical relevance of improved microbleed detection by susceptibility-weighted magnetic resonance imaging. Stroke 2011;42:1894-900.

13. Hoshi T, Kitagawa K, Yamagami H, Furukado S, Hougaku H, Hori M. Relations of serum high-sensitivity C-reactive protein and interleukin-6 levels with silent brain infarction. Stroke 2005;36:768-72.

14. Li J, Zhao Y, Mao J. Association between the extent of white matter damage and early cognitive impairment following acute ischemic stroke. Exp Ther Med 2017;13:909-12.

15. Cordonnier C, Al-Shahi Salman R, Wardlaw J. Spontaneous brain microbleeds: systematic review, subgroup analyses and standards for study design and reporting. Brain 2007;130:1998-2003.

16. Shams S, Martola J, Granberg T, Li X, Shams M, Fereshtehnejad SM, Cavallin L, Aspelin P, Kristoffersen-Wiberg M, Wahlund LO. Cerebral microbleeds: different prevalence, topography, and risk factors depending on dementia diagnosis -- the Karolinska Imaging Dementia Study. AJNR Am J Neuroradiol 2015;36:661-6.
17. Poels MM, Zaccai K, Verwoert GC, Vernooij MW, Hofman A, van der Lugt A, Witteman JC, Breteler MM, Mattace-Raso FU, Ikram MA. Arterial stiffness and cerebral small vessel disease: the Rotterdam Scan Study. Stroke 2012;43:2637-42.

18. Poels MM, Vernooij MW, Ikram MA, Hofman A, Krestin GP, van der Lugt A, Breteler MM. Prevalence and risk factors of cerebral microbleeds: an update of the Rotterdam Scan Study. Stroke 2010;41:S103-6.

19. Chung PW, Park KY, Kim JM, Shin DW, Ha SY. Carotid artery calcification is associated with deep cerebral microbleeds. Eur Neurol 2014;72:60-3.

20. Poels MM, Ikram MA, van der Lugt A, Hofman A, Niessen WJ, Krestin GP, Breteler MM, Vernooij MW. Cerebral microbleeds are associated with worse cognitive function: the Rotterdam Scan Study. Neurology 2012;78:326-33

21. Gustavsson AM, Stomrud E, Abul-Kasim K, Minthon L, Nilsson PM, Hansson O, Nägga K. Cerebral microbleeds and white matter hyperintensities in cognitively healthy elderly: a cross-sectional cohort study evaluating the effect of arterial stiffness. Cerebrovasc Dis Extra 2015;5:41-51.

22. Miwa K, Tanaka M, Okazaki S, Furukado S, Sakaguchi M, Kitagawa K. Relations of blood inflammatory marker levels with cerebral microbleeds. Stroke 2011;42:3202-6.

23. Schrag M, McAuley G, Pomakian J, Jiffry A, Tung S, Mueller C, Vinters HV, Haacke EM, Holshouser B, Kido D, Kirsch WM. Correlation of hypointensites insusceptibility weighted images to tissue hitology in dementia patients with cerebral amyloid angiopathy: a postmortem MRI study. Acta Neuropathol 2010;119:291-302.

24. Koh SH, Park CY, Kim MK, Lee KY, Kim J, Chang DI, Kim HT, Kim SH. Microbleeds and free active MMP-9 are independent risk factors for neurological deterioration in acute lacunar stroke. Eur J Neurol 2011;8:158-64.

25. Pantoni L. Cerebral small vessel disease: from pathogenesis and clinical characteristics to therapeutic challenges. Lancet Neurol 2010;9:689-701.

26. Piao MS, Lee JK, Park CS, Ryu HS, Kim SH, Kim HS. Early activation of matrix metalloproteinase- 9 is associated with bloodbrain barrier disruption after photothrombotic cerebral ischemia in rats. Acta Neurochir (Wien) 2009;151:1649-53.

27. Jin R, Yang G, Li G. Molecular insights and therapeutic targets for blood-brain barrier disruption in ischemic stroke: critical role of matrix metalloproteinases and tissue-type plasminogen activator. Neurobiol Dis 2010;38:376-85.

28. Schrag M, McAuley G, Pomakian J, Jiffry A, Tung S, Mueller C, Vinters HV, Haacke EM, Holshouser B, Kido D, Kirsch WM. Correlation of hypointensities in susceptibility-weighted images to tissue histology in dementia patients with cerebral amyloid angiopathy: a post-mortem MRI study. Acta Neuropathol 2010;119:291-302.

29. Kim BJ, Lee SH. Cerebral microbleeds: their associated factors, radiologic findings, and clinical implications. Stroke 2013;15:153-63. 